\title{
Tea and Coffee Consumption and MRSA Nasal Carriage
}

Eric M. Matheson, MD, MS

Arch G. Mainous III, PbD

Charles J. Everett, $P b D$

Dana E. King, MD, MS

Department of Family Medicine, Medical University of South Carolina, Charleston, South Carolina
Conflicts of interest: authors report none.

\section{CORRESPONDING AUTHOR}

Eric M. Matheson, MD, MS Department of Family Medicine Medical University of South Carolina 295 Calhoun St

Charleston, South Carolina 29425

Matheson@musc.edu

\begin{abstract}
PURPOSE Hot tea and coffee have been found to have antimicrobial properties. The purpose of this study was to determine whether the consumption of tea, coffee, or both is associated with less frequent nasal carriage of methicillin-resistant Staphylococcus aureus (MRSA).
\end{abstract}

METHODS We performed a secondary analysis of data from the 2003-2004 National Health and Nutrition Examination Survey to investigate the relationship between the consumption of coffee, hot tea, cold tea, and soft drinks, and MRSA nasal carriage among the noninstitutionalized population of the United States.

RESULTS An estimated 2.5 million persons (1.4\% of the population) were MRSA nasal carriers. In an adjusted logistic regression analysis controlling for age, race, sex, poverty-income ratio, current health status, hospitalization in the past 12 months, and use of antibiotics in the past month, individuals who reported consuming hot tea were one-half as likely to have MRSA nasal carriage relative to individuals who drank no hot tea (odds ratio $=0.47 ; 95 \%$ confidence interval, 0.31-0.71). Similarly, individuals who reported consuming coffee had about a one-half reduction in the risk of MRSA nasal carriage relative to individuals who drank no coffee (odds ratio $=0.47 ; 95 \%$ confidence interval, $0.24-0.93$ ).

CONCLUSIONS Consumption of hot tea or coffee is associated with a lower likelihood of MRSA nasal carriage. Our findings raise the possibility of a promising new method to decrease MRSA nasal carriage that is safe, inexpensive, and easily accessible.

Ann Fam Med 2011;9:299-304. doi:10.1370/afm.1262.

\section{INTRODUCTION}

$\mathrm{I}$ nfection with methicillin-resistant Stapbylococcus aureus (MRSA) is a major source of morbidity and mortality in the United States. In 2005, more than 278,000 Americans were hospitalized for MRSA-related infections, and there were more than 6,500 MRSA-related deaths. ${ }^{1}$ Studies have suggested that nasal carriage of MRSA may significantly increase the risk of a MRSA infection, although this finding has not been universal. ${ }^{2-4}$

In an effort to both prevent and treat MRSA, researchers have examined the antimicrobial effects of several commonly consumed plants and plant extracts. ${ }^{5-7}$ Two of the most promising and ubiquitous are tea and coffee $^{8-10}$ Tea has been found to have antimicrobial activity against several types of pathogenic bacteria, including Vibrio cholerae, Escherichia coli, Shigella, Salmonella, and S aureus. ${ }^{11-13}$ Like tea, coffee appears to have antimicrobial properties against Escherichia coli, Salmonella, and $S$ aureus. ${ }^{10,14}$ Both in vivo and in vitro studies have demonstrated that tea or tea-based extracts have antimicrobial properties when applied topically., What remains unclear is whether tea and coffee have systemic antimicrobial activity when consumed orally as beverages.

The purpose of this study was to evaluate the relationship between oral consumption of tea and coffee, and MRSA nasal carriage in a nationally representative sample of Americans. 


\section{METHODS}

\section{Study Design and Participants}

We analyzed data from the 2003-2004 National

Health and Nutrition Examination Survey (NHANES) sample ${ }^{16}$ a nationally representative sample of the noninstitutionalized population of the United States. ${ }^{17}$ The NHANES uses a complex survey design that allows for the determination of population estimates. To enable these estimates, the survey uses sampling weights that are calculated by taking into account the unequal probabilities of selection resulting from sample design, nonresponse, and planned oversampling of minorities.

The NHANES sampling procedure consists of 4 stages. Stage 1 entails selection of primary sampling units (PSUs), which are mostly single counties or, in a few cases, groups of contiguous counties with probability proportional to a measure of size. In stage 2, the PSUs are divided into segments (generally city blocks or their equivalent). In stage 3, households within each segment are listed and a sample is randomly drawn. In geographic areas where the proportion of age, ethnic, or income groups selected for oversampling is high, the probability of selection for those groups is greater than in other areas. In stage 4, individuals are chosen to participate in NHANES from a list of all persons residing in selected households. The individuals are drawn at random within designated domains of age, sex, and race/ethnicity. Each individual is assigned a sample weight, a measure of the number of people in the population represented by that individual in NHANES, reflecting the unequal probability of selection, adjustment for nonresponse, and adjustment to individual population controls. The 30 PSUs included in the 2003-2004 NHANES were selected from a frame of all US counties, using the 2000 census data.

More information on the methods of the 20032004 NHANES survey, including laboratory assessments, can be found elsewhere. ${ }^{16}$ Nasal carriage of $S$ aureus was assessed in all participants aged 1 year or older, but many of the other variables were not included for individuals younger than age 2. Consequently, we included in our study only participants aged 2 years old or older.

\section{Assessment of MRSA Nasal Carriage}

Nasal cultures were collected and plated on mannitol salt agar (MSA). The MSA plates were incubated at $37^{\circ} \mathrm{C}$ for 48 hours. Mannitol-fermenting colonies were selected and subcultured to plates with trypticase soy agar containing 5\% sheep blood, that is, blood agar plates (BAPs), and incubated at $37^{\circ} \mathrm{C}$ overnight. MSA plates with little or no grow th were reincubated at $37^{\circ} \mathrm{C}$ overnight, and plates with non-mannitol-fermenting growth were held at room temperature. The plates were reexamined the next day, and any yellow or gold colonies were subcultured to BAPs.

Overnight cultures on BAPs were first screened using Staphaurex, a rapid latex kit for the identification of $S$ aureus (Remel Laboratories, Lenexa, Kansas). A tube coagulase test using rabbit plasma with EDTA was then performed both on Staphaurex-negative isolates from BAPs with morphology consistent with $S$ aureus and on Staphaurex-positive isolates with morphology inconsistent with $S$ aureus. Staphaurex-positive isolates and Staphaurex-negative, tube coagulase-positive isolates were identified as $S$ aureus and saved for further testing.

\section{Determination of Methicillin Resistance}

$S$ aureus isolates were screened for methicillin resistance by the disk diffusion method. Overnight cultures from BAPs were plated on Mueller-Hinton agar, and a 1- $\mu$ g oxacillin disk was placed on the inoculated plate. Zone diameters were measured and recorded after a 24 -hour incubation at $37^{\circ} \mathrm{C}$; on the basis of the results, isolates were classified as sensitive ( $\geq 13 \mathrm{~mm})$, intermediate (11$12 \mathrm{~mm})$, or resistant $(\leq 10 \mathrm{~mm})$. For the purposes of this study, $S$ aureus isolates with a zone diameter of $10 \mathrm{~mm}$ or less were considered MRSA and all other Stapbylococcus isolates were considered methicillin sensitive (MSSA).

\section{Beverage Consumption}

Beverages evaluated were hot tea, iced tea, coffee, soda consumed during the summer, and soda consumed during the remainder of the year as ascertained with 6 questions. The first question was, "How many cups of hot tea, caffeinated or decaffeinated, did you drink (over the past 12 months)?" The second question was, "How many glasses of iced tea, caffeinated or decaffeinated, did you drink (over the past 12 months)?" The third question was "How many cups of coffee, caffeinated or decaffeinated, did you drink (over the past 12 months)?" Responses to these 3 questions were categorized as (1) none or (2) less than 1 cup a month or more for coffee, tea, and iced tea. Participants were also asked, "Over the past 12 months, did you drink soft drinks, soda, or pop?" Those who answered yes were then asked, "How often did you drink soft drinks, soda, or pop in the summer?" and subsequently asked, "How often did you drink soft drinks, soda, or pop during the rest of the year?" Because of the very small number of individuals who did not consume any soft drinks, answers for soft drink consumers were classified as (1) never to 2 to 3 times per month or (2) 1 to 6 times per week or more.

\section{Control Variables}

We evaluated numerous variables previously associated with MRSA nasal carriage as potential confounding variables in the relationship between MRSA carriage 
and beverage consumption. They included age, race, sex, poverty status, health status, recent hospital stay, and recent antibiotic use.

Age was categorized into groups (2-6, 7-19, 20-64, and $\geq 65$ years old). Race/ethnicity was classified as non-Hispanic white, non-Hispanic black, or Hispanic. We did not include individuals categorized as Other Race or Multiracial in our study because there were too few with MRSA (unweighted $n=4$ ). Poverty status was determined using the poverty-income ratio. This ratio is calculated by dividing a family's income by a poverty threshold income that is based on family size. ${ }^{18}$ The poverty threshold is determined each year by the US Office of Management and Budget. Povertyincome ratios ranged up to 5, with values of less than 1 indicating poverty. Current health status was based on self-report and was classified as either (1) excellent, very good, or good, or (2) fair or poor. Recent hospitalization was determined by participants' response to the question, "During the past 12 months were you a patient in a hospital overnight? Do not include an overnight stay in the emergency room."

Recent antibiotic use was evaluated in the NHANES by examining the individual's prescription medications. Participants were asked to bring the containers for all the prescription medications taken during the past month and to report any medications taken for which the container was not available. These medications were then matched by trained survey interviewers to an annually updated comprehensive database of all prescription drugs in the US market.

\section{Missing Data}

For the purposes of this analysis, we excluded individuals who were missing data on any variable. A total of 3,172 individuals were missing data. Of these individuals, the majority were missing data on coffee consumption $(2,654)$, tea consumption $(2,658)$ or povertyincome ratio (449).

\section{Analysis}

We used SUDAAN software (Research Triangle Institute, Research Triangle Park, North Carolina) for all analyses to account for the complex sample design used in NHANES. The analyses incorporated both the stratification and clustering aspects of the sampling design. The proper weighting procedures include adjustments for basic probability of selection and nonresponse. Because minorities were oversampled, and the sampling design was complex, we used sampling weights provided by the NHANES to compute population estimates based on weighted parameter estimates and standard errors.

We computed descriptive statistics for the study sample. Because there were relatively small numbers of MRSA carriers within each beverage consumption group, it was necessary to use a binary categorization (drinker or nondrinker) for the beverage groups to gain a standard error of less than $30 \%$, conforming to recommendations of the National Center for Health Statistics (which conducts NHANES) for small sample sizes. We performed a $\chi^{2}$ analysis to determine that none of the standard errors exceeded $30 \%$ on population estimates, as recommended by the Center. We computed unadjusted and adjusted logistic regression models to determine the odds ratios (ORs) and 95\% confidence intervals (CIs) for MRSA nasal carriage according to beverage consumption. In addition to hot tea and coffee, iced tea and soft drink consumption were examined to determine if the antimicrobial properties of hot tea and coffee differed from that of other types of beverages. The adjusted models controlled for age, race/ethnicity, sex, poverty-income ratio, current health status, hospitalization in the past year, and antibiotic use in the past month. A statistical level of $P$ $<.05$ was considered significant for all analyses.

\section{RESULTS}

The sample included 5,555 individuals, which is equivalent to a weighted sample size of $182,100,249$. Their characteristics are shown in Table 1.

Overall, $1.4 \%$ of the individuals in the sample had nasal carriage with MRSA. An estimated 88.6 million persons (48.6\% of the population) reported consuming hot tea, and 93.7 million (51.4\% of the population) reported consuming no hot tea over the past month. An estimated 110.7 million (60.8\% of the population) reported consuming coffee, and 71.4 million $39.2 \%$ of the population) reported consuming no coffee over the past month.

In an unadjusted logistic regression model (Table 2 ), individuals who consumed hot tea had a slightly more than one-half reduction in the likelihood of MRSA nasal carriage relative to their counterparts who reported consuming no hot tea $(\mathrm{OR}=0.47 ; 95 \% \mathrm{CI}$, 0.31-0.70). Furthermore, after adjusting for age, race/ ethnicity, sex, poverty-income ratio, current health status, hospitalization in the past 12 months, and use of antibiotics in the past month, individuals who reported consuming hot tea still had a lower likelihood of MRSA nasal carriage $(\mathrm{OR}=0.47 ; 95 \% \mathrm{CI}, 0.31-0.71)$.

Coffee consumption was also associated with a one-half reduction in the odds of MRSA nasal carriage, but only in the adjusted logistic regression model $(\mathrm{OR}=0.47$; 95\% CI, 0.24-0.93) (Table 2).

The findings were similar for combined coffee and tea intake. The odds of MRSA nasal carriage were lower for individuals drinking both beverages com- 


\section{Table 1. Characteristics of the Sample (Unweighted N = 5,555; Population Estimate $=182,100,249$ )}

\begin{tabular}{|c|c|c|c|}
\hline Characteristic & $\begin{array}{c}\text { Participants, } \\
\%\end{array}$ & Characteristic & $\begin{array}{c}\text { Participants, } \\
\%\end{array}$ \\
\hline Age, y & & Nasal carriage status & \\
\hline $2-6$ & 6.1 & MRSA & 1.4 \\
\hline $7-19$ & 18.6 & MSSA & 26.5 \\
\hline $20-64$ & 61.8 & No Staphylococcus & 72.1 \\
\hline$\geq 65$ & 13.5 & Coffee & \\
\hline Race/ethnicity & & None & 39.2 \\
\hline Non-Hispanic white & 76.2 & $<1$ cup per month or more & 60.8 \\
\hline Non-Hispanic black & 11.6 & Hot tea & \\
\hline Hispanic & 12.2 & None & 51.4 \\
\hline Sex & & $<1$ cup per month or more & 48.6 \\
\hline Male & 46.8 & Coffee or hot tea & \\
\hline Female & 53.2 & None & 26.8 \\
\hline Poverty-income ratio & & $<1$ cup per month or more & 73.2 \\
\hline$<1$ & 14.3 & of either & \\
\hline$\geq 1$ & 85.7 & Iced tea & \\
\hline Current health status & & None & 34.0 \\
\hline Good to excellent & 85.7 & $<1$ cup per month or more & 66.0 \\
\hline Fair to poor & 14.3 & $\begin{array}{l}\text { Soft drinks during the } \\
\text { summer }\end{array}$ & \\
\hline $\begin{array}{l}\text { Hospitalized in the } \\
\text { past } 12 \text { months }\end{array}$ & & $\begin{array}{l}\text { Never to 2-3 times per } \\
\text { month }\end{array}$ & 24.4 \\
\hline Yes & 9.2 & 1-6 times per week or more & 75.6 \\
\hline $\begin{array}{l}\text { No } \\
\text { Use of antibiotics in }\end{array}$ & 90.8 & $\begin{array}{l}\text { Soft drinks during the rest } \\
\text { of the year }\end{array}$ & . \\
\hline $\begin{array}{l}\text { the past month } \\
\text { Yes }\end{array}$ & 5.6 & $\begin{array}{l}\text { Never to } 2-3 \text { times per } \\
\text { month }\end{array}$ & 30.9 \\
\hline No & 94.4 & $1-6$ times per week or more & 69.1 \\
\hline
\end{tabular}

ally representative sample of adults and children in the United States, extend the previous findings of in vitro and topical antimicrobial activity of tea and coffee to a systemic effect. The results suggest that consumption of coffee and hot tea may modify the risk of MRSA nasal carriage and potentially MRSA infection.

The mechanism behind this possible effect of coffee and tea consumption on MRSA carriage is not completely understood. There is increasing evidence to suggest that both coffee and tea have antimicrobial characteristics. ${ }^{8,10,17}$ Although the exact mechanisms through which the antimicrobial properties of these beverages are exerted remain to be elucidated, a few compounds have garnered particular attention. In the case of coffee, particular attention has focused on the potential antibacterial properties of trigonelline, glyoxal, methylglyoxal, and diacetyl. ${ }^{10,19}$ For tea, attention has focused on the antimicrobial properties of tannic acid and catechins. ${ }^{8,20,21}$ In addi- pared with individuals drinking neither tea nor coffee, in both the unadjusted logistic regression model $(\mathrm{OR}=0.41 ; 95 \% \mathrm{CI}, 0.20-0.81)$ and the adjusted logistic regression model $(\mathrm{OR}=0.33 ; 95 \% \mathrm{CI}, 0.16-0.70)$.

In contrast to the associations found for hot tea and coffee, we did not find any statistically significant relationship between iced tea or soft drink consumption and MRSA nasal carriage (Table 2).

Because fewer children than adults consume coffee and tea, we conducted a separate analysis to examine the association between coffee and tea consumption and MRSA nasal carriage among individuals aged 18 years or older. The results were similar to those in the entire sample in adjusted analyses, for hot tea $(\mathrm{OR}=0.53 ; 95 \% \mathrm{CI}, 0.15-0.80)$, for coffee $(\mathrm{OR}=0.49$; $95 \% \mathrm{CI}, 0.23-1.04)$, and for the combination of hot tea and coffee $(\mathrm{OR}=0.35 ; 95 \% \mathrm{CI}, 0.15-0.80)$.

\section{DISCUSSION}

The results of this study suggest a lower likelihood of MRSA nasal carriage among individuals who drink hot tea, coffee, or both. These findings, based on a nation- tion, consumption of both coffee and tea decrease iron absorption, which may prove important because iron is critical for the growth of $S$ aureus. ${ }^{22,23}$

Although most of the data on the antimicrobial properties of coffee and hot tea have come from in vitro experiments, a few clinical trials have examined the effect of hot tea and coffee in vivo. In one study conducted by Fujii et al ${ }_{1}^{9}$ bedridden patients with MRSA-infected decubitus ulcers underwent debridement of their ulcers with either green tea or normal saline. After 1 month, the patients whose ulcers were treated with green tea debridement experienced a marked decrease in ulcer severity, and MRSA had disappeared in one-half. In the patients treated with normal saline debridement, ulcer severity was unchanged, and all of the ulcers were still infected with MRSA.

In another study, 24 elderly patients with cerebrovascular disease and MRSA-positive sputum were randomized and treated with either tea catechin extracts or saline as a control, each given by a nebulizer 3 times daily, for 4 weeks. ${ }^{15}$ After 1 week, 7 of the 12 patients in the tea-treated group had a decrease in MRSA concentration in their sputum, compared 


\section{Table 2. Logistic Regression Models Showing Odds of MRSA Nasal Carriage by Beverage Consumption}

\begin{tabular}{|c|c|c|}
\hline Beverage Consumption & $\begin{array}{l}\text { Unadjusted OR } \\
\qquad(95 \% \mathrm{Cl})\end{array}$ & $\begin{array}{c}\text { Adjusted }^{\mathrm{a}} \text { OR } \\
(95 \% \mathrm{Cl})\end{array}$ \\
\hline \multicolumn{3}{|l|}{ Coffee } \\
\hline None & 1.00 & 1.00 \\
\hline$<1$ cup per month or more & $0.53(0.28-1.02)$ & $0.47(0.24-0.93)$ \\
\hline \multicolumn{3}{|l|}{ Hot tea } \\
\hline None & 1.00 & 1.00 \\
\hline$<1$ cup per month or more & $0.47(0.31-0.70)$ & $0.47(0.31-0.71)$ \\
\hline \multicolumn{3}{|l|}{ Coffee or hot tea } \\
\hline None & 1.00 & 1.00 \\
\hline$<1$ cup per month or more of either & $0.41(0.20-0.81)$ & $0.33(0.16-0.70)$ \\
\hline \multicolumn{3}{|l|}{ Iced tea } \\
\hline None & 1.00 & 1.00 \\
\hline$<1$ cup per month or more & $0.81(0.39-1.68)$ & $0.88(0.39-1.99)$ \\
\hline \multicolumn{3}{|l|}{ Soft drinks during the summer } \\
\hline Never to 2-3 times per month & 1.00 & 1.00 \\
\hline 1-6 times per week or more & $0.85(0.44-1.66)$ & $0.97(0.48-1.96)$ \\
\hline \multicolumn{3}{|l|}{ Soft drinks during the rest of the year } \\
\hline Never to 2-3 times per month & 1.00 & 1.00 \\
\hline 1-6 times per week or more & $1.07(0.57-1.99)$ & $1.28(0.68-2.42)$ \\
\hline
\end{tabular}

imputation, which may have increased our standard error. Third, we could not be certain when individuals last consumed coffee or tea. Finally, because an exact understanding about the biochemical constituents in hot tea and coffee that may be associated with protection against MRSA remains to be elucidated, we had to speculate on a primary theory and several alternative theories. The effect, however, appears to be robust.

Although not a specific limitation, it is important to note that the larger question of the importance of MRSA nasal carriage has yet to be resolved. Even though several studies have suggested such carriage increases the risk of systemic infection with MRSA, others have not. ${ }^{2-4}$ Given this debate, the benefits of any treatment for MRSA nasal carriage should be carefully balanced against the risk.

At present, the only way to treat MRSA nasal carriage is with antibiotics that have proven only partially successful at longterm eradication. Our findings of reduced odds of MRSA nasal carriage among tea and coffee drinkers raise the possibility of a promising new alternative to antibiotics that is safe, inexpensive, and easily accessible.

To read or post commentaries in response to this article, see it online at http://www.annfammed.org/cgi/content/full/9/4/299.

Key words: Methicillin-resistant Staphylococcus aureus; MRSA; tea; coffee; disease prevention; carriers; disease reservoirs; colonization

Submitted October 14, 2010; submitted, revised, January 11, 2011; accepted February 4, 2011.

Funding support: The National Health and Nutrition Examination Survey (NHANES) is conducted and supported by the National Center for Health Statistics (NCHS), which is part of the Centers for Disease Control and Prevention (CDC).

Disclaimer: This manuscript was prepared using the NHANES public access data set and does not necessarily reflect the opinions or views of the NCHS or the CDC.

\section{References}

1. Klein E, Smith DL, Laxminarayan R. Hospitalizations and deaths caused by methicillin-resistant Staphylococcus aureus, United States, 1999-2005. Emerg Infect Dis. 2007;13(12):1840-1846.

2. Safdar N, Bradley EA. The risk of infection after nasal colonization with Staphylococcus aureus. Am J Med. 2008;121(4):310-315.

3. Honda H, Krauss MJ, Coopersmith CM, et al. Staphylococcus aureus nasal colonization and subsequent infection in intensive care unit patients: does methicillin resistance matter? Infect Control Hosp Epidemiol. 2010;31(6):584-591. the study with missing values rather than using multiple 
4. Sarikonda KV, Micek ST, Doherty JA, Reichley RM, Warren D, Kollef $\mathrm{MH}$. Methicillin-resistant Staphylococcus aureus nasal colonization is a poor predictor of intensive care unit-acquired methicillin-resistant Staphylococcus aureus infections requiring antibiotic treatment. Crit Care Med. 2010;38(10):1991-1995.

5. Al-Habib A, Al-Saleh E, Safer AM, Afzal M. Bactericidal effect of grape seed extract on methicillin-resistant Staphylococcus aureus (MRSA). J Toxicol Sci. 2010;35(3):357-364.

6. Mahomoodally MF, Gurib-Fakim A, Subratty AH. Screening for alternative antibiotics: an investigation into the antimicrobial activities of medicinal food plants of Mauritius. J Food Sci. 2010;75(3): M173-M177.

7. Tsao SM, Hsu CC, Yin MC. Garlic extract and two diallyl sulphides inhibit methicillin-resistant Staphylococcus aureus infection in BALB/ CA mice. J Antimicrob Chemother. 2003;52(6):974-980.

8. Yam TS, Shah S, Hamilton-Miller JMT. Microbiological activity of whole and fractionated crude extracts of tea (Camellia sinensis), and of tea components. FEMS Microbiol Lett. 1997;152(1):169-174.

9. Fujii M, Ohrui T, Sato T, Sato T, Sato N, Sasaki H. Green tea for decu bitus in bedridden patients. Geriatr Gerontol Int. 2003;3(4):208-211.

10. Daglia M, Papetti A, Grisoli P, et al. Isolation, identification, and quantification of roasted coffee antibacterial compounds. J Agric Food Chem. 2007;55(25):10208-10213.

11. Bandyopadhyay D, Chatterjee TK, Dasgupta A, Lourduraja J, Das tidar SG. In vitro and in vivo antimicrobial action of tea: the commonest beverage of Asia. Biol Pharm Bull. 2005;28(11):2125-2127.

12. Yamada $\mathrm{H}$, Tateishi M, Harada $\mathrm{K}$, et al. A randomized clinical study of tea catechin inhalation effects on methicillin-resistant Staphylococcus aureus in disabled elderly patients. I Am Med Dir Assoc. 2006;7(2):79-83.

13. Cho YS, Schiller NL, Oh KH. Antibacterial effects of green tea polyphenols on clinical isolates of methicillin-resistant Staphylococcus aureus. Curr Microbiol. 2008;57(6):542-546.

14. Rufián-Henares JA, de la Cueva SP. Antimicrobial activity of coffee melanoidins - a study of their metal-chelating properties. J Agric Food Chem. 2009;57(2):432-438.

15. Yamada H, Ohashi $K$, Atsumi $T$, et al. Effects of tea catechin inhalation on methicillin-resistant Staphylococcus aureus in elderly patients in a hospital ward. J Hosp Infect. 2003;53(3):229-231.

16. Centers for Disease Control and Prevention (CDC); National Center for Health Statistics (NCHS). National Health and Nutrition Examination Survey Data. Hyattsville, MD: US Dept of Health and Human Services. http://www.cdc.gov/nchs/nhanes/nhanes2003-2004/ nhanes03 04.htm. Accessed Dec 6, 2010.
17. Kumar J, Muntner P, Kaskel FJ, Hailpern SM, Melamed ML. Prevalence and associations of 25-hydroxyvitamin D deficiency in US children: NHANES 2001-2004. Pediatrics. 2009;124(3):e362-e370.

18. US Census Bureau. How the census bureau measures poverty. 2009. http://www.census.gov/hhes/www/poverty/about/overview/ measure.html. Accessed Dec 6, 2010.

19. Almeida AA, Farah A, Silva DA, Nunan EA, Glória MB. Antibacterial activity of coffee extracts and selected coffee chemical compounds against enterobacteria. J Agric Food Chem. 2006;54(23):8738-8743.

20. Gradisar H, Pristovsek P, Plaper A, Jerala R. Green tea catechins inhibit bacterial DNA gyrase by interaction with its ATP binding site. J Med Chem. 2007;50(2):264-271.

21. Akiyama H, Fujii K, Yamasaki O, Oono T, Iwatsuki K. Antibacterial action of several tannins against Staphylococcus aureus. J Antimicrob Chemother. 2001;48(4):487-491.

22. Skaar EP, Humayun M, Bae T, DeBord KL, Schneewind O. Ironsource preference of Staphylococcus aureus infections. Science. 2004;305(5690):1626-1628

23. Temme EH, Van Hoydonck PG. Tea consumption and iron status. Eur J Clin Nutr. 2002;56(5):379-386.

24. Hakim IA, Weisgerber UM, Harris RB, Balentine D, van Mierlo CA, Paetau-Robinson I. Preparation, composition and consumption patterns of tea-based beverages in Arizona. Nutr Res. 2000;20(12):1715-1724

25. Lin SL, Liu EL, Mau JM. Effect of different brewing methods on antioxidant properties of steaming green tea. LWT-Food Sci Technol. 2008;41(9):1616-1623.

26. Shimoda M, Shigematsu H, Shiratsuchi H, Osajima Y. Comparison of volatile compounds among different grades of green tea and their relations to odor attributes J Agric Food Chem. 1995;43(6):1621-1625

27. Olszewski WL, Grzelak I, Ziolkowska A, Engeset A. Effect of local hyperthermia on lymph immune cells and lymphokines of normal human skin. J Surg Oncol. 1989;41(2):109-116.

28. Terndrup TE, Allegra JR, Kealy JA. A comparison of oral, rectal, and tympanic membrane-derived temperature changes after ingestion of liquids and smoking. Am J Emerg Med. 1989;7(2):150-154. 